\title{
Current perspectives on the role of the pharmacist in heart failure management
}

This article was published in the following Dove Press journal: Integrated Pharmacy Research and Practice

\section{Judy WM Cheng}

Department of Pharmacy Practice, Massachusetts College of Pharmacy and Health Sciences (MCPHS) University, Brigham and Women's Hospital, Boston, MA, USA
Correspondence: Judy WM Cheng Department of Pharmacy Practice, MCPHS University, Brigham and Women's Hospital, 179 Longwood Avenue, Boston, MA 02115-5896, USA $\mathrm{Tel}+\mathrm{I} 6177322868$

Fax +I 6177322244

Email judy.cheng@mcphs.edu

\begin{abstract}
Pharmacists play an important role within a multidisciplinary health care team in the care of patients with heart failure (HF). It has been evaluated and documented that pharmacists providing medication reconciliation especially during transition of care, educating patients on their medications, and providing collaborative medication management lead to positive changes in the patient outcomes, including but not limited to decreasing in hospitalizations and readmissions. It is foreseeable that pharmacist roles will continue to expand as new treatment and innovative care are developed for HF patients. I reviewed published role of pharmacists in the care of HF patients. MEDLINE and Current Content database (both from 1966 - December $31,2017)$ were utilized to identify peer-reviewed clinical trials, descriptive studies, and review articles published in English using the following search terms: pharmacists, clinical pharmacy, HF, and cardiomyopathy. Citations from available articles were also reviewed for additional references. Preliminary search revealed 31 studies and 55 reviews. They were further reviewed by title and abstract as well as full text to remove irrelevant articles. At the end, 24 of these clinical trials and systematic reviews are described in the following text and Table 1 summarizes 16 pertinent clinical trials. Some roles that are currently being explored include medication management in patients with mechanical circulatory support for end-stage HF, where pharmacokinetics and pharmacodynamics of medications can change, medication management in ambulatory intravenous diuretic clinics, and comprehensive medication management in patients' home settings. Pharmacists should continue to explore and prospectively evaluate their role in the care of this patient population, including documenting their interventions, and impact to economic and patient outcomes.
\end{abstract}

Keywords: pharmacists, heart failure

\section{Introduction}

Heart failure (HF) affects 5.7 million Americans and post a cost of \$30.7 billion each year to the nation, including cost of health care services, medications, and missed days of work. ${ }^{1}$ Over the past few decades, pharmacotherapy including angiotensinconverting enzyme inhibitors (ACEIs), angiotensin receptor blockers and neprilysin inhibitors (ARNIs), beta-blockers and aldosterone antagonists, to name a few, has been demonstrated to improve survival in HF with reduced ejection fraction. However, HF morbidity and mortality remain high. It was documented that up to $44 \%$ of patients after a recent HF hospital admission will be readmitted in 6 months. ${ }^{2}$ Optimally managing these patients require a multidisciplinary team approach attuned to many aspects of care. ${ }^{3}$ Pharmacists are important members of this multidisciplinary team and can 
contribute significantly in optimizing patients' complicated drug regimen. ${ }^{4}$ This article described published evidence evaluating the different roles of pharmacists in the care of HF patients, the potential impact in patient outcomes to different extent, as well as discuss future perspective of such role.

\section{Method}

MEDLINE and Current Content database (both 1966 to December 31, 2017) were utilized to identify peer-reviewed clinical trials, descriptive studies, and review articles published in English using the following search terms: pharmacists, clinical pharmacy, HF, and cardiomyopathy. Citations from available articles were also reviewed for additional references. Our preliminary search revealed 31 studies and 55 reviews. Because pharmacists' role is diverse and varied in different clinical settings, we did not limit our search to certain kind of interventions. The manuscript aims to describe all documented interventions. The search results were further reviewed by title and abstracts as well as full text to remove irrelevant articles. At the end, 24 of these clinical trials and systematic reviews are described in the following text and Table 1 summarizes 16 pertinent clinical trials.

\section{Clinical pharmacists' role in HF care}

Pharmacists responsibilities in the care of $\mathrm{HF}$ are diverse and well documented in the literature. ${ }^{4}$ Although each practice setting (inpatient vs outpatient) provides unique opportunity for different types of clinical pharmacist contributions/interventions, there are a few important aspects of services that appear to be consistent performed across different practice settings. These include medication reconciliation and patient medications education; providing pharmacotherapeutic recommendations and monitoring (including drug initiation, adjustment, and monitoring); early identification and prevention of adverse drug reactions and interactions; and improving medication adherence, access to medications and transition of care.

\section{Medication reconciliation and patient medication education}

Medication reconciliation is a process of comparing patient's medication orders to all of the medications that they may have been taking, in order to avoid errors such as omission, duplications, dosing errors, and interactions, especially when there is a transit of patient care from one setting to another (from home to hospital admission, from hospital to discharge home, from long-term rehabilitation to home, etc).

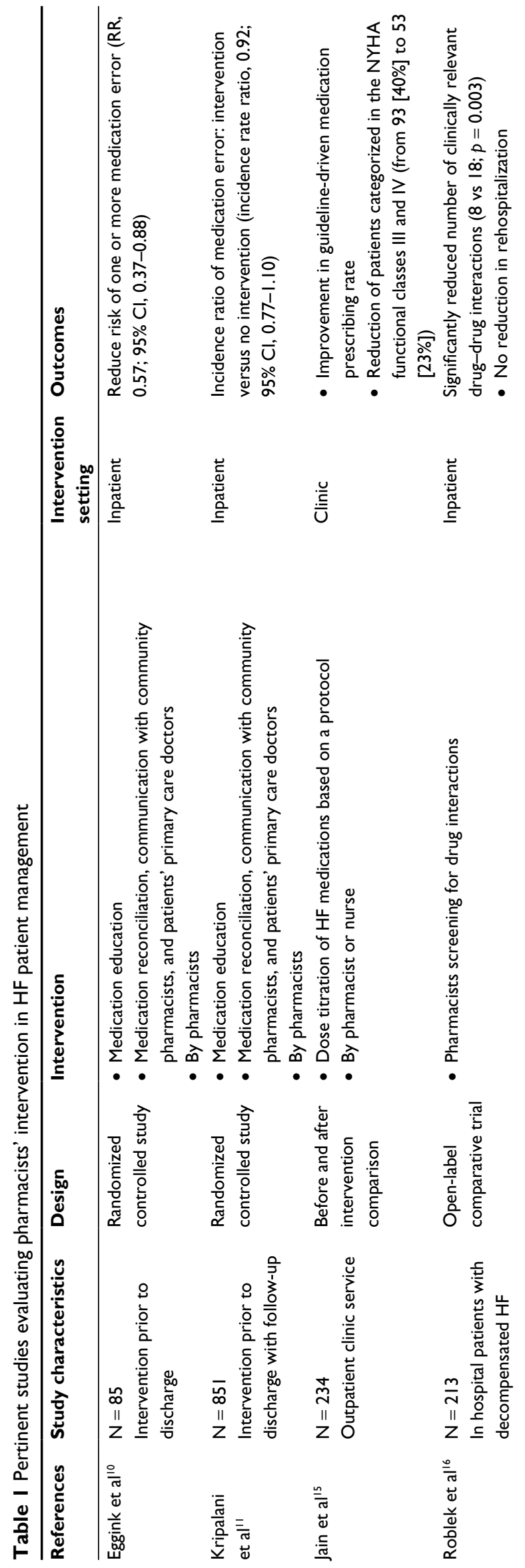




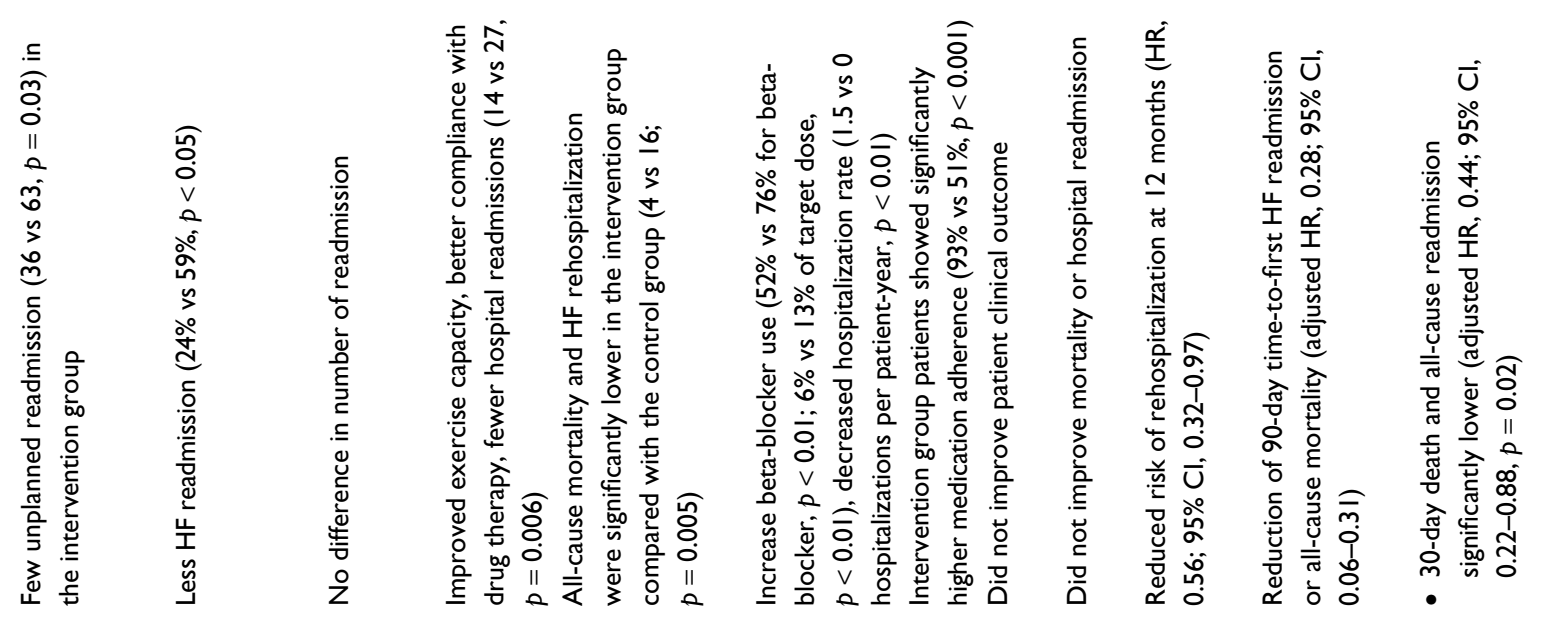

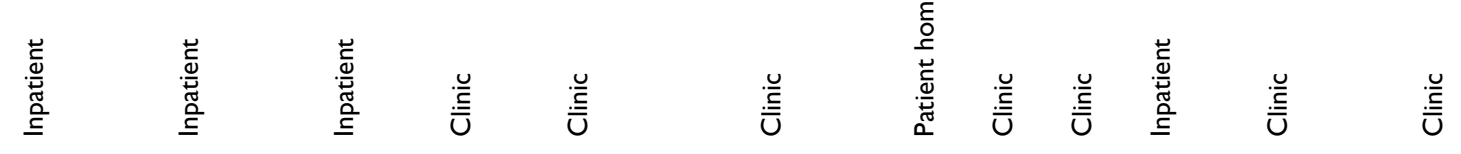
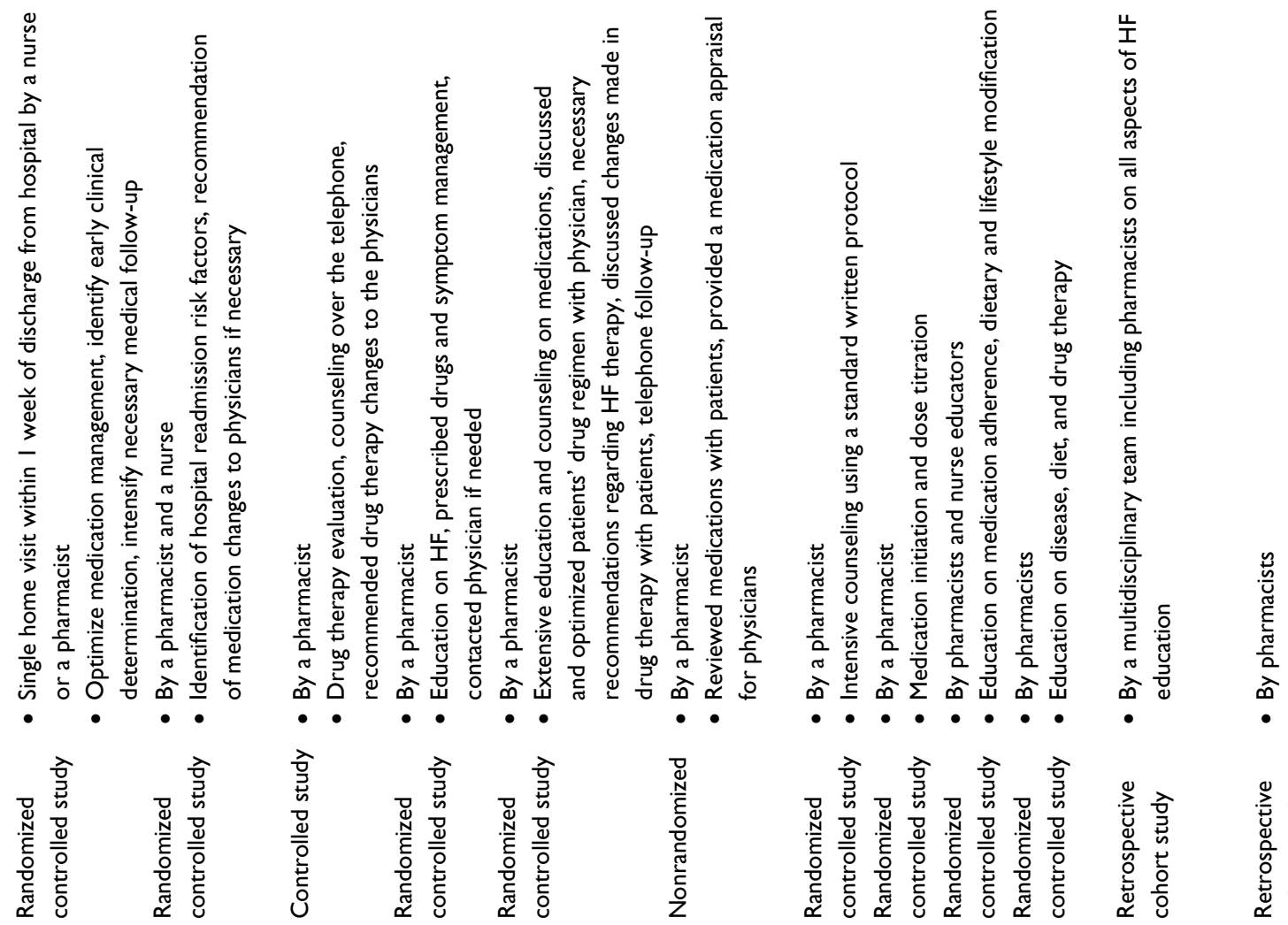

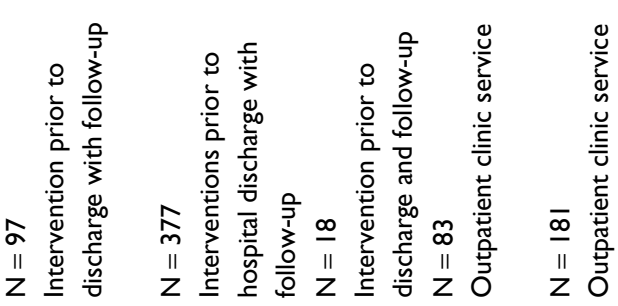
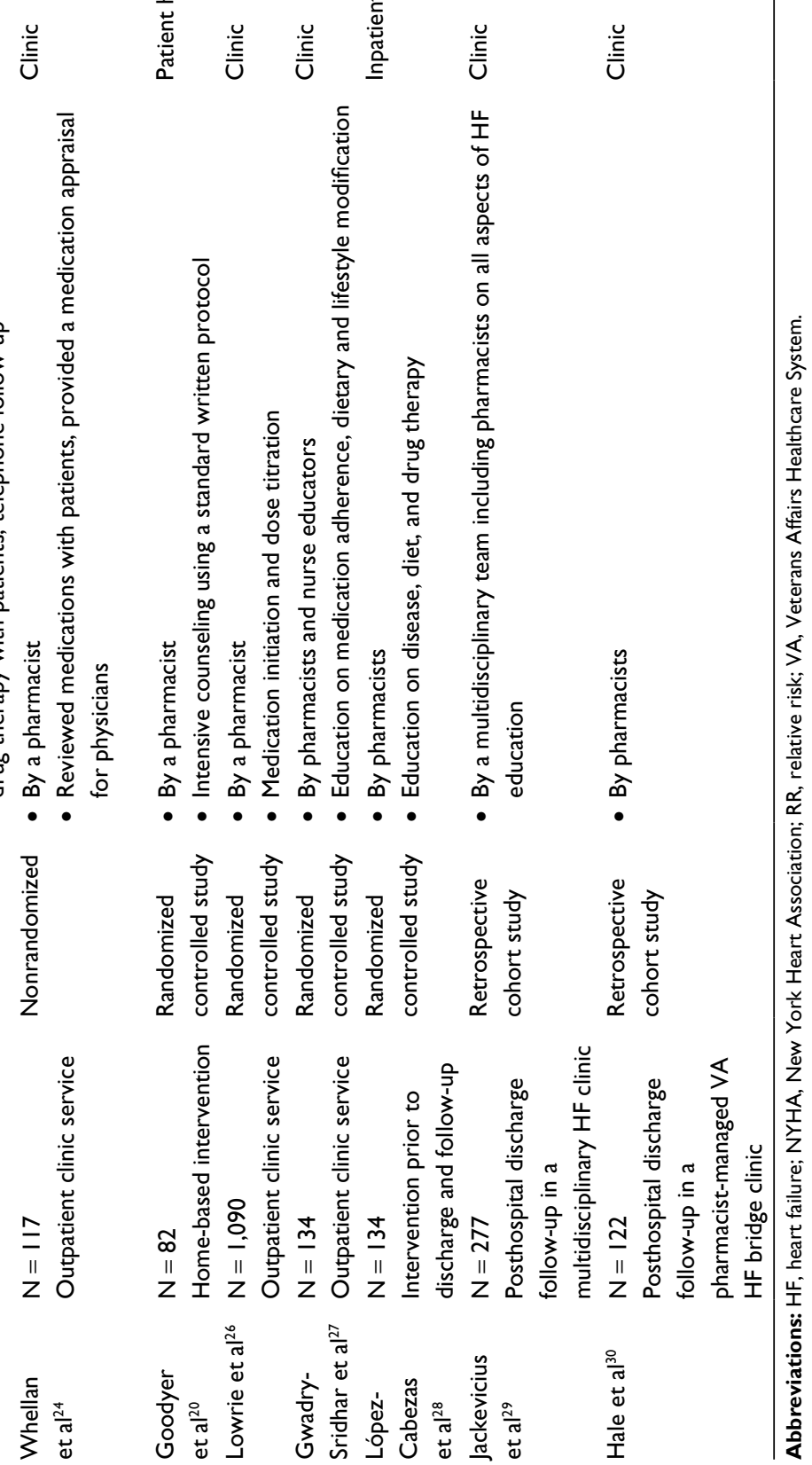

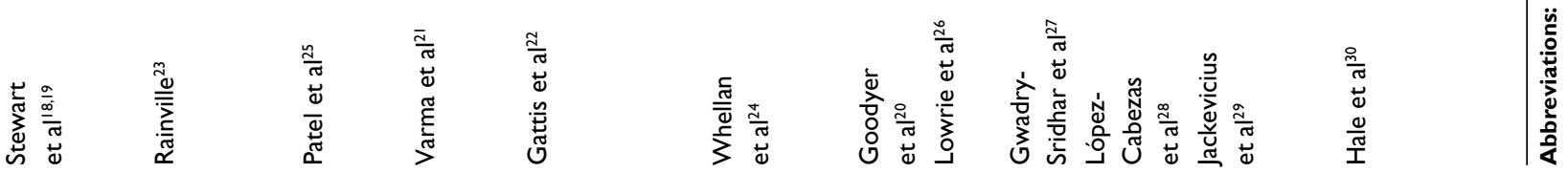


Medication reconciliation is a critical component of care to ensure medication safety and is part of the US Joint Commission National Patient Safety Goals. It is well described in literatures that during transition of care from one setting to another, such as discharge from hospital to home, patients are most susceptible to medication errors related to inaccurate medication histories. ${ }^{5-7}$ HF patients have complex medical regimens, require frequent dosage changes and modifications, and usually have multiple other comorbidities. These factors increase the likelihood for medication discrepancies. Medication reconciliations are performed by many different health care professionals (physicians, nurses, etc). Studies have found that when pharmacists were involved in medication reconciliation, there was a significant reduction in medication errors and improvement of patient knowledge retention because of the ability to access medication records from other settings, and a more comprehensive approach in obtaining medication history. ${ }^{8,9}$ Eggink et al evaluated the clinical pharmacist impact on prescribing errors when performing discharge medication reconciliation for $\mathrm{HF}$ patients. ${ }^{10}$ Patients are randomized to receiving medication reconciliation at discharge from pharmacists versus usual care. In addition to providing medication reconciliation service, clinical pharmacists also review medications with patients and communicate with patients' community pharmacists and primary physicians regarding their discharge medications. The primary study endpoint included combination of a number of prescribing errors in discharge medications and medication postdischarge. Usual care group has 44 patients, whereas pharmacist intervention group has 41. Sixty-eight percent of patients in the usual care group had at least one prescribing discrepancy, whereas $39 \%$ in the pharmacist intervention group had at least one prescribing discrepancy (relative risk [RR] 0.57; 95\% CI, 0.37-0.88). This study demonstrated the impact of a clinical pharmacist in reducing prescribing errors when providing medication reconciliation during the transition of care process upon patient discharge.

The Pharmacist Intervention for Low Literacy in Cardiovascular Disease study evaluated impact of pharmacist medication reconciliation in 30-day posthospital discharged medication errors for acute coronary syndrome and HF patients. ${ }^{11}$ In this study, patients were randomized to either usual care or pharmacist intervention. Services provided by a pharmacist included admission medication history, medication reconciliation at admission and discharge, and educating patients on their medications on discharge. Overall, of the 851 patients enrolled, $432(50.8 \%)$ had one or more clinically important medication errors at 30 days $(22.9 \%$ were considered serious and $1.8 \%$ life threatening). Adverse drug events were experienced by 258 patients (30.3\%). Pharmacist intervention did not significantly change the per-patient number of clinically important medication errors (unadjusted incidence rate ratio, $0.92 ; 95 \% \mathrm{CI}, 0.77-1.10)$. However, there was a trend of greater benefits from medication reconciliation in patients with low health literacy and nonsignificant benefit in patients with high health literacy.

\section{Pharmacotherapeutic recommendations and monitoring}

Over the last several decades, clinical evidence has been established supporting the use of certain pharmacologic management of HF. Drug therapy for chronic HF with reduced ejection fraction management centers predominantly around ACEI or angiotensin receptor blockers (ARBs), ARNI, betablockers and aldosterone antagonists for improvement of mortality, and ivabradine or digoxin to aid reduction of hospitalization. However, despite abundant evidence, it is well documented that these therapies are not optimally prescribed in actual clinical practice. ${ }^{12}$ The Acute Decompensated Heart Failure National Registry collected case data from $\sim 40,000$ patients with decompensated HF patients across the USA. Data showed that on hospital discharge, only 54\% of eligible patients received ACEI, $12 \%$ received ARB, and $72 \%$ received beta-blockers. ${ }^{13}$ These data have significantly improved compared to those previously reported and have shown improvement of use of evidence-based therapies. Understandably, some of these patients may have a more severe stage of disease and may not be able tolerate these evidence-based mortality improving regimens due to lower blood pressure or poorer renal function. However, the use of evidence-based therapy (ACEI and beta-blockers), especially newer agents such as ARNI or ivabradine, in those at highest risk of hospital readmission has not been thoroughly studies and may still have room for improvement.

The Enhanced Feedback for Effective Cardiac Treatment trial, a population-based cohort study that evaluated 9,942 patients hospitalized with HF in Ontario, Canada, ${ }^{14}$ reviewed that evidence-based pharmacotherapy was suboptimally used in patients at high risk of death as compared with low risk (ACEI or ARB [adjusted HR, 1.61; 95\% CI, 1.49-1.74] and beta-blockers [HR, 1.80; 95\% CI, 1.60-2.01]). At discharge, ACEI was prescribed in $81 \%, 73 \%$, and $60 \%$ in patients at low, intermediate, and high risk of death, respectively; when including ARBs, prescribing rates were $86 \%, 80 \%$, and $65 \%$ at discharge, respectively, and prescribing rates were $40 \%$, $33 \%$, and $24 \%$ at discharge for beta-blockers, respectively. 
Jain et al assessed the impact of a protocol-driven HF clinic (staffed by nurses and pharmacists) in improving guideline-driven use of pharmacotherapy and patient symptoms. ${ }^{15}$ Of the 234 patients in the study, 127 (57\%) of them were receiving none or only one guideline-driven evidencebased therapy during their first clinic visit. This was reduced to 25 patients $(11 \%)$ after being managed by the clinic for 1 year. In addition, the improvement in prescription rates was accompanied by significant up-titration of dose. The proportion of patients on "medium" or "high" doses increased for beta-blockers from $43(18 \%)$ to $134(57 \%)$ and for ACEI/ ARB from 129 (55\%) to 201 (86\%). Symptoms improvement was demonstrated by the reduction of patients categorized in the New York Heart Association functional classes III and IV (from 93 [40\%] to 53 [23\%]). This study, however, did not describe specific impact made by a nurse versus a pharmacist.

\section{Identification and prevention of adverse drug reactions and interactions}

Roblek et al examined the impact of pharmacist intervention on reduction of clinically significant drug-drug interactions (DDIs) in hospitalized patients with ADHF management. ${ }^{16}$ Patients were randomized to pharmacist intervention or standard care group. Patients in the intervention group received alerts about clinically significant DDIs. Primary endpoint was number of DDIs at discharge and secondary endpoints were rehospitalization or death within 6 months of discharge. Of the 213 patients enrolled, 66 clinically relevant DDIs were identified. The number of patients with clinically significant DDIs was significantly lower at discharge in the intervention group compared with the control group, respectively (8 vs $18 ; p=0.003$ ). Over a 6-month follow-up period, 11 control and 9 intervention patients were rehospitalized or died ( $p$ $=0.2$ ). This study showed that pharmacist intervention can significantly reduce the number of patients with clinically relevant DDIs but may not translate to improvement in longer term outcome.

Dempsey et al described the patient population served in a new innovative Ambulatory Cardiac Triage, Intervention, and Education infusion unit and to document the prevalence of comorbidities and drug therapy-related issues (DRIs) in order to define the most effective role of a pharmacist in the unit. ${ }^{17}$ Patients' medical and medication profiles were reviewed. DRIs were identified and classified. Sixty patients were interviewed. Most prevalent cardiac comorbidities were hypertension (73\%) and hyperlipidemia (62\%). Top three noncardiac comorbidities included chronic kidney disease $(60 \%)$, diabetes $(50 \%)$, and obesity $(35 \%)$. DRI prevalence was reported as: 1) needs of an additional/alternative therapy (untreated indication [37] or suboptimal therapeutic choice [46]); 2) wrong drug (major DDIs [90], contraindication [11], or duplicate therapy [1]); 3) suboptimal dosing (17); 4) dose exceeds recommended maximum (9); and 5) adverse drug reaction (93). In 63 (22\%) of the DRIs, recommendations were made by the pharmacist to modify the regimen. The study indicated that the prevalence of DRIs is high even among HF patients managed in a subspecialty cardiovascular practice. Pharmacists can contribute significantly in resolving DRI.

Overall, pharmacists can play a significant role in identifying adverse drug events and drug interactions in patients who have heavy medication burdens.

\section{Improving medication adherence, access to medications, and transition of care}

Perhaps, the most extensively researched and documented area in pharmacists' role in HF patient management was the evaluation of pharmacists' involvement in improving medication adherence and access during transition of care in an "outpatient" or "postdischarge" clinic setting.

Stewart et al evaluated the effect of health care professional intervention at patients' home for 97 patients who were recently discharged from the hospital with HF. ${ }^{18}$ The intervention was delivered by a team of nurses and pharmacists who provided one home visit within 1-week posthospital discharge. Interventions focused on medication regimens optimization, identification of early symptom deterioration, and arrangement of medical follow-up if necessary. Pharmacists also evaluated patients' medication knowledge and their adherence. Patients showing poor medication knowledge and/or nonadherence to medication regimen received further education, phone reminder to take medications, and a medication administration aid (eg, pillbox). Referrals were also made to a community pharmacist for regular medication review. Patients in the intervention group had fewer unplanned hospital readmissions (36 vs 63, $p=0.03$ ) and less out-of-hospital deaths ( 1 vs $5, p=0.11$ ).

In 1999, the same group of investigators reported the results for an extended 12-month follow-up of all surviving patients. ${ }^{19}$ Results continued to demonstrate fewer unplanned readmissions, out-of-hospital deaths, and days of hospitalization for the patients who received interventions in their homes.

Goodyer et al conducted a study to evaluate if intensive medication counseling provided by pharmacists to elderly patients with chronic HF can impact HF outcomes. ${ }^{20}$ Patients 
$(\mathrm{n}=82)$ were randomized to receive a 3-month medication counseling program or no counseling. A "standard written protocol" was used by all pharmacists to perform the counseling. Adherence was measured by a pill count, and medication knowledge was assessed using a questionnaire. At baseline, mean adherence was $49 \%$ in the control group as compared with $61 \%$ for the intervention group $(p=0.98)$. After the program, adherence was significantly improved in the intervention group (93\% vs $51 \%, p<0.001)$, so is medication knowledge $(p<0.001)$. Patients in the intervention group demonstrated significant improvement in 6-minute exercise test at the end of the study, as well as improvement of leg edema, while the control group did not.

Varma et $\mathrm{al}^{21}$ assessed the impact of a structured pharmaceutical care program for 83 patients with HF aged over 65 years. Patients were randomized into an interventional group, where they received verbal education from a pharmacist on the disease state, medications and symptoms management of $\mathrm{HF}$, and a take home' printed booklet. Patients were required to self-monitor using diary cards. Physicians and community pharmacists reviewed these cards and mailed them to the researchers. From these assessments, patients who received interventions demonstrated better adherence with drug therapy and improved exercise tolerance compared with those in the control group. Over the 12 months of this study, the intervention group also had fewer hospital admissions compared to the control group (14 vs 27 readmission, $p=0.006$ ).

Gattis et al conducted the Pharmacist in Heart Failure Assessment Recommendation and Monitoring Study to examine the benefits of having a pharmacist as part of the HF management team on ambulatory patient outcomes. ${ }^{22}$ In the intervention group $(n=90)$, pharmacists provided in-depth medication education and counseling. The pharmacist worked collaboratively with patient's physician to implement changes to the patient's HF drug therapy. Primary endpoint was the combination of all-cause mortality and nonfatal HF events (including emergency department visits and hospitalizations for HF). Four events occurred in the intervention group $(\mathrm{n}=$ $90)$ versus 16 events in the control group $(n=91)(p=0.005)$. Although, effect on all-cause mortality was not significant, nonfatal HF events were significantly reduced. The investigators suggested that close patient follow-up led to the early recognition and management of signs and symptoms of fluid overload, thus preventing deterioration. In addition, patients in the intervention group were more likely to receive target ACE inhibitor doses. ${ }^{22}$

The impact of pharmacists' interventions on the functional health status of $377 \mathrm{HF}$ patients and their rate of hospital readmissions were evaluated at an acute care facility. ${ }^{23}$ Other health care professionals were blinded to the allocation of interventions. Patients were randomized to medication education provided by pharmacists or routine care. The pharmacist reviewed the pharmacotherapy and pathology of HF with patients and their caregiver; monitored patients' weight and worked on cardiovascular risk modifications; provided a patient information brochure, medication organizer, weight log booklet, and a video tape; and recommended medication changes to the physician when necessary. There was a significant reduction in HF hospital readmissions in the intervention group as compared with the control group $(24 \%$ vs $59 \%, p<0.05)$ over the 12-month period. In addition, there was a significantly longer time to readmission for the patients in the intervention group. ${ }^{23}$

Whellan et $\mathrm{al}^{24}$ evaluated the impact of an HF disease management program, the Duke Heart Failure Program. This study assessed the benefits of beta-blocker use and the cost to the health care system. This program involved many different health professionals. The specific role of pharmacists included patient medication education and collaboration with physicians to optimize patient medication regimen. One hundred and seventeen patients were enrolled. Patients were followed up for $\sim 5$ months. The pharmacist, with the help of the nurse, emphasized weight monitoring and instructed patients when to contact a DHFP nurse in the event of experiencing worsening symptoms. The program demonstrated increased usage of beta-blocker and achievement of target doses (52\% vs $76 \%$ for beta-blocker, $p<0.01 ; 6 \%$ vs $13 \%$ of target dose, $p<0.01)$. The program also demonstrated reduction in hospitalization rates ( 1.5 vs 0 hospitalizations per patient-year, $p<0.01)$, but the number of ambulatory clinic visits significantly increased (4.3 vs 9.8 clinic visits per patient-year, $p<0.01)$. The doses of ACE inhibitors used also increased, although not significantly. ${ }^{24}$

The role of pharmacists as part of a pilot HF program to help prevent exacerbations and hospitalizations among HF patients was evaluated. ${ }^{25}$ Pharmacists in the intervention group evaluated and optimized patient medication therapy and provided telephone medication counseling and recommendations of drug therapy changes to physicians. In this pilot study, there was no difference in the number of hospitalizations between the intervention and control groups. This study, however, was limited by a small sample size $(\mathrm{n}=18)$.

Lowrie et al randomized 1,090 HF patients from 87 primary care practices to pharmacist interventions versus usual care. ${ }^{26}$ Pharmacists reviewed patient medication regimen and 
recommended initiating ACE or ARB or beta-blocker therapy if patients were not already receiving them. Dose adjustment was also made accordingly. The primary outcome (composite death or hospital admission for worsening HF) occurred in $35.8 \%$ of patients in the intervention group and $35.4 \%$ in the usual care group (HR, 0.97; 95\% CI, 0.83-1.14; $p=0.72)$. The investigators concluded that pharmacist-led collaborative intervention in primary care setting resulted in modest improvements in prescribing of disease-modifying medications but did not improve patient clinical outcomes. However, it is important to note that the study population had a high baseline percentage of being on evidence-based guidelinedirected therapy. Therefore, there may not be enough power to detect any difference, if exists.

Gwadry-Sridhar et al performed a randomized controlled pilot study, where 134 patients with HF with reduced ejection fraction were randomized into the intervention group, where they received medication adherence education and dietary and lifestyle modification provided by a team of pharmacist and a nurse educator or usual care. ${ }^{27}$ The intervention did not improve mortality. However, hospital readmission and emergency department visits were reduced. Patients reported significant improvement in quality of life measured by the Minnesota Living with Heart Failure Questionnaire. This study, however, did not differentiate the specific impact on patient outcome between the pharmacists and the nurse educators.

López-Cabezas et al randomized 134 patients hospitalized for $\mathrm{HF}^{28}$ into usual care or intervention, which included disease state education and diet and drug therapy review at hospital discharge. Patient received follow-up phone call at 2, 6, and 12 months postdischarge. At 12 months of follow-up, 32.9\% fewer patients in the intervention group were admitted again versus the control group (HR, 0.56; 95\% CI, 0.32-0.97).

Jackevicius et al evaluated the impact of a multidisciplinary HF clinic on 90-day hospital readmission rates and all-cause mortality in patients recently discharged from a hospital for HF management. ${ }^{29}$ Patients discharged with a primary HF diagnosis who attended the HF postdischarge follow-up clinic in 2010-2012 were compared with controls from 2009. During 6 follow-up visits, patients were seen by a physician assistant, a clinical pharmacist specialist, and a case manager, with a cardiologist overseeing the care. Interventions performed at the clinic included optimizing therapy, identifying/managing HF etiology/precipitating factors, education on medication and medication adherence, and dose titration. The primary outcome was 90-day HF readmission. Among the 277 patients (144 clinic, 133 control) included in the study, $7.6 \%$ of patients from the follow-up clinic and $23.3 \%$ of patients from the control group were readmitted for HF within 90 days (adjusted HR, 0.17; 95\% CI, 0.07-0.41; $p<0.001$ ). Follow-up clinic patients also had lower 90-day time-to-first HF readmission or all-cause mortality $(9.0 \%$ vs $28.6 \%$; adjusted HR, 0.28; 95\% CI, 0.06-0.31). This outpatient clinical follow-up program was associated with a significant reduction in 90-day HF readmissions.

Hale et al assessed the impact of a pharmacist-managed $\mathrm{HF}$ bridge clinic in a veteran population. ${ }^{30} \mathrm{HF}$ patients hospitalized from November 2010 to August 2013 were identified. Retrospective chart review was conducted of $122 \mathrm{HF}$ patients seen at bridge clinic compared with 122 randomly selected HF patients not seen at this clinic (usual care). Primary endpoint was 90-day all-cause readmission and death. Secondary outcomes were 30-day all-cause readmission and death, time to first postdischarge followup, and first all-cause readmission. Bridge clinic patients were at higher baseline risk of readmission and death; other characteristics were similar. The 90-day death and all-cause readmission trended lower in bridge clinic patients (adjusted HR, 0.64; 95\% CI, 0.40-1.02; $p=0.06$ ). Time to first follow-up was shorter in bridge clinic patients ( $11 \pm 6$ vs $20 \pm 23$ days; $p<0.001)$; time to first all-cause readmission trended longer ( $40 \pm 20$ vs $33 \pm 25$ days; $p=0.11)$. The 30 -day death and all-cause readmission was significantly lower in bridge clinic patients (adjusted HR, 0.44; 95\% CI, 0.22-0.88; $p=0.02$ ). In veteran patients hospitalized for HF, pharmacistmanaged HF bridge clinic significantly reduced the time to initial follow-up compared with usual care.

Published literatures consistently demonstrated that pharmacist involvement in transition of care and providing early postdischarge follow-up, either individually or as part of a team, reduce hospital readmission.

\section{Impact on patient outcomes}

Published studies have described different roles of pharmacist in the care of HF patients, in different settings and with different outcome measurements. Some of the studies included small number of patients, and there was not always a comparative control group. Therefore, meta-analysis and systematic reviews may help us to better determine the composite impact of these services to patient outcomes.

Holland et al performed a systematic review of 30 randomized controlled trials evaluating the impact of multidisciplinary team interventions on HF patients' outcome in terms of all-cause hospital admission, mortality, and HF hospital admission, in both hospital and community 
settings. ${ }^{31}$ Multidisciplinary interventions reduced all-cause hospital admission (RR, 0.87; 95\% CI, 0.79-0.95), although there was significant heterogeneity found among studies reviewed $(p=0.002)$. All-cause mortality was also reduced (RR, 0.79; 95\% CI, 0.69-0.92) as was HF admission (RR, $0.70 ; 95 \% \mathrm{CI}, 0.61-0.81)$. These results varied little with sensitivity analyses.

Koshman et al also performed another systematic review to characterize the role of pharmacist in the care of patients with HF. ${ }^{32}$ Twelve randomized controlled studies were identified. The interventions were categorized as pharmacist-directed care or pharmacist collaborative care. Overall, pharmacist care (both directed and collaboration) was associated with significant reductions in the rate of all-cause hospitalizations (11 studies [2,026 patients]) (OR, $0.71 ; 95 \%$ CI, 0.54-0.94) and HF hospitalizations (11 studies [1,977 patients]) (OR, 0.69; 95\% CI, 0.51-0.94), and a nonsignificant reduction in mortality (12 studies [2,060 patients]) (OR, 0.84; 95\% CI, 0.61-1.15). Pharmacist collaborative care led to greater reductions in the rate of HF hospitalizations (OR, 0.42; 95\% CI, 0.24-0.74) than pharmacist-directed care (OR, 0.89; 95\% CI, 0.68-1.17). The authors concluded that pharmacist care in the management of patients with HF greatly reduces the risk of all-cause and HF hospitalization, especially when it was performed in collaboration with a multidisciplinary team.

Kang et al performed a systematic review of studies evaluating pharmacists' involvement in care of HF patients. ${ }^{33}$ After evaluation of the strength of the body of evidence, 14 studies were meta-analyzed. The evidence was not strong enough to determine the effects of pharmaceutical care on patient-centered outcomes. All-cause hospitalization (OR, $0.74 ; 95 \%$ CI, 0.58-0.94) was reduced with pharmacists' involvement in care, and the prescription rates of ACEI (OR 1.43; 95\% CI, 1.07-1.91) and beta-blockers (OR 1.92; 95\% CI, 1.24-2.96) were significantly higher compared with the usual care group. The investigators suggested that the lack of improvement in patient-centered outcomes could be explained by the diversity of pharmaceutical care delivered by different pharmacists and the heterogeneity of patient populations or clinical settings. The investigators suggested that a standardized consensus of the guidelines for pharmaceutical care service should be considered to better able to evaluate impact of pharmaceutical care in the future.

\section{Potential/future role of HF pharmacists Management of mechanical circulatory support}

Advances in mechanical circulatory support, such as the use of ventricular-assisted devices (VADs), have prolonged survival for patients with end-stage HF. VADs are currently used as a bridge to heart transplantation, a bridge to recovery of cardiac function after cardiothoracic surgery, or as palliative care. VADs, however, come with complications including bleeding and thrombosis, infections, arrhythmias, multiple organ failure, and right ventricular failure. Patients with VADs have unique pharmacotherapeutic needs. Because VAD technology evolves quickly, evidence available in managing this patient population is still sparse compared to other areas of HF management. Therefore pharmacists' expertise in pharmacology and therapeutics can play a unique role in helping these patients in optimizing their care. Jennings et al described the development of a clinical pharmacy service specifically for patients with left VAD. ${ }^{34}$ Out of 400 interventions documented, the most common type was change in medication regimens $(33 \%)$, followed by initiating evidencebased HF therapy (31\%), discontinuing therapy (18\%), ordering a monitoring laboratory test $(12 \%)$, and changing to a more optimal therapy $(6 \%)$. The most common reasons for pharmacist intervention were treatment of a disease or condition that was not optimally managed on present therapy (36\%), followed by dose correction (17\%), improvement of monitoring of drug therapy (13\%), and prevention of adverse drug reaction/DDIs (11\%). This area of highly specialized clinical pharmacy practice is at an early pilot stage and will continue to evolve as newer generations of mechanical heart devices are developed and utilization of these devices continue to increase. Pharmacists may obtain special trainings and develop a unique role in managing pharmacotherapy in this patient population.

\section{Outpatient HF infusion clinic}

The rate of hospital readmissions for ADHF is a major benchmark statistic for third-party payers including Medicare and private insurers. Many health care institutions tried to design innovative strategies to prevent and reduce hospital admissions. A small pilot study has demonstrated that planned intravenous diuretic therapy administered in an outpatient setting may reduce HF hospital admissions and 30-day readmissions. ${ }^{35}$ Another study evaluated a multidisciplinary team approach (including a pharmacist) in the use of intravenous diuretic therapy in management of HF and volume overload in an outpatient unit. ${ }^{36}$ Sixty patients with chronic HF and clinical evidence of worsening congestion were admitted to the clinic to receive a bolus and 3-hour IV infusion of furosemide. Outcomes in terms of urine output during the visit, weight loss at 24 hour, and hospitalization and mortality at 30 days were similar across patients with varying maintenance diuretic doses. Hospitalization was 
reported by nurse practitioner evaluation upon arrival to the clinic as imminent for $52.8 \%$ of patients; however, the observed rate of all-cause hospitalization was only $31.7 \%$ at 30 days with no death, indicating a $21.1 \%$ reduction in hospitalization rate. Many institutions across the US have interest in developing these clinics. Such setting provides another opportunity for pharmacists to collaborate with a health care team to optimize patients' medication therapy. It also allows pharmacists to work directly with patients in terms of medication reconciliation, educating them on their medications, and improving medication adherence.

\section{Discussion}

Based on the studies reviewed, three trials have demonstrated that pharmacist participation in the care of HF patients reduced medication errors; ${ }^{10,11,16}$ four trials showed improvement in prescribing of guideline-driven medication therapy and medication adherence, ${ }^{15,20,21,24}$ two demonstrated improvement in symptoms, ${ }^{15,21}$ and among those that evaluated hospital readmission as endpoint, eight demonstrated reduction in rehospitalization, ${ }^{18,19,21-24,28-30}$ including two that also demonstrated reduction in mortality, ${ }^{22,29}$ whereas four did not demonstrate any improvement in rehospitalization. ${ }^{16,25-27}$ The differences in impact of pharmacist interventions observed is unclear but may be explained by limitations of individual studies, including size of study and number of patients enrolled, whether pharmacists intervene by themselves or as part of a disease management team, and the specific intervention performed and whether such intervention was standardized, especially if multiple pharmacists perform the same interventions. It is also important to note that pharmacist trainings may vary and HF patients have complex medication regimen, and the specific pharmacist performing the intervention may affect the outcome. Based on the three systematic analysis published, they concluded that pharmacists overall, when combining the results of these studies, improve readmission. ${ }^{31-33}$

Over years, medical and therapeutic advances have been made with respect to HF and its management. Despite these advances, therapeutic challenges still exist and prognosis of these patients is still poor. These patients have high requirement for health care resources. Pharmacists play an important role in the management of the HF patients through medication reconciliation, patient education, and collaborative medication management efforts to optimize therapy. Studies have shown that pharmacists, when actively engaged in these efforts in various settings, may decrease medication errors and improve medication use and prescribing and symptoms.
Larger prospective randomize trial and standardize pharmacist intervention may be required to confirm the actual impact on clinical outcomes such as rehospitalization and mortality. The American College of Clinical Pharmacy and the HF Society of American published an opinion paper in support of clinical pharmacists' involvement in HF patient care. The opinion paper also made recommendations on minimum training required that will potentially allow clinical pharmacists to effectively engage in HF patient care. HF patients have complicated medication regimen. In addition to having a entry level pharmacy practice degree, pharmacists serving HF patient population will require postgraduate trainings, including but not limited to clinical residencies specializing in cardiovascular diseases, critical care or organ transplant, or clinical research fellowship in corresponding area. ${ }^{37}$ In the USA, there are also specialized credentialing processes that certify pharmacists' capability in caring for this patient population. ${ }^{37}$ In addition, many states have regulations governing pharmacists entering a collaborative practice agreement with physicians or a health care team to provide drug therapy management, so everyone's unique role in the team will be more defined. ${ }^{37}$ Financial strategies were also proposed to support clinical pharmacy services within a multidisciplinary team. ${ }^{37}$ As the health care system continues to bring forward innovative approach to care in order to minimize the HF disease burden, pharmacists will have even more ways and expanded role to contribute to the care of HF patients.

\section{Conclusion}

Management of HF patients can benefit from a multidisciplinary team approach. HF patients not only require often complex medication regimen but also require other lifestyle modification and close medical follow-up to ensure optimal clinical outcomes. Pharmacists can play an important role within a multidisciplinary health care team in care of patients with HF. It has been evaluated and documented that pharmacists providing medication reconciliation especially during transition of care, educating patients on their medications, and providing collaborative medication management lead to positive changes in the patient outcomes, including reduction in medication errors and drug interactions, improvement in medication adherence, and improvement of use of guidelinedriven therapy. Some studies also demonstrated reduction in hospital readmission. Pharmacists should continue to explore, define, and standardize the intervention and continue to prospectively evaluate their role in the care of this patient population, designing study with enough power to confirm 
the impact on economic and clinical outcomes including hospitalization and mortality.

\section{Disclosure}

The author reports no conflicts of interest in this work.

\section{References}

1. Centers for Disease Control and Prevention. Heart failure fact sheet. Available from: https://www.cdc.gov/dhdsp/data_statistics/fact_sheets/ fs_heart_failure.htm. Accessed August 29, 2017.

2. Krumholz HM, Parent EM, Tu N, et al. Readmission after hospitalization for congestive heart failure among Medicare beneficiaries. Arch Intern Med. 1997;157(1):99-104.

3. Takeda A, Taylor SJ, Taylor RS, Khan F, Krum H, Underwood M. Clinical service organization for heart failure. Cochrane Database Syst Rev. 2012;9:CD002752.

4. Stough WG, Patterson JH. Role and value of clinical pharmacy in heart failure management. Clin Pharmacol Ther. 2017;102(2):209-212.

5. LaPointe NM, Jollis JG. Medication errors in hospitalized cardiovascular patients. Arch Intern Med. 2003;163(12):1461-1466.

6. Bobb A, Gleason K, Husch M, Feinglass J, Yarnold PR, Noskin GA. The epidemiology of prescribing errors: the potential impact of computerized prescriber order entry. Arch Intern Med. 2004;164(7):785-792.

7. Gleason KM, Groszek JM, Sullivan C, Rooney D, Barnard C, Noskin GA. Reconciliation of discrepancies in medications histories and admission orders of newly hospitalized patients. Am J Health Syst Pharm. 2004;61(16):1689-1695.

8. Christensen M, Lundh A. Medication review in hospitalized patients to reduce morbidity and mortality. Cochrane Database Syst Rev. 2013;2:CD008986.

9. Ryan R, Santesso N, Hill S, Lowe D, Kaufman C, Grimshaw J. Consumer-oriented interventions for evidence based prescribing and medicines use: an overview of systemic reviews. Cochrane Database Syst Rev. 2011;5:CD007768.

10. Eggink RN, Lenderink AW, Widdershoven JW, van den Bemt PM. The effect of a clinical pharmacist discharge service on medication discrepancies in patients with heart failure. Pharm World Sci. 2010;32(6):759-766.

11. Kripalani S, Roumie CL, Dalal AK, et al; PILL-CVD (Pharmacist Intervention for Low Literacy in Cardiovascular Disease) Study Group. Effect of a pharmacist intervention on clinically important medication errors after hospital discharge: a randomized trial. Ann Intern Med. 2012;157(1):1-10.

12. Rodgers JE, Gattis-Stough W. Underutilization of evidence-based therapies in heart failure: the pharmacist's role. Pharmacotherapy. 2007;27(4 Pt 2):18S-28S

13. Fonarow GC, Yancy CW, Heywood JT; ADHERE Scientific Advisory Committee, Study Group, and Investigators. Adherence to heart failure quality-of-care indicators in U.S. hospitals: analysis of the ADHERE registry. Arch Intern Med. 2005;165(13):1469-1477.

14. Lee DS, Tu JV, Juurlink DN, et al. Risk-treatment mismatch in the pharmacotherapy of heart failure. JAMA. 2005;294(10):1240-1247.

15. Jain A, Mills P, Nunn LM, et al. Success of a multidisciplinary heart failure clinic for initiation and up-titration of key therapeutic agents. Eur J Heart Fail. 2005;7(3):405-410.

16. Roblek T, Deticek A, Leskovar B, et al. Clinical pharmacist intervention reduces clinically relevant drug-drug interactions in patients with heart failure: a randomized, double-blind controlled trial. Int J Cardiol. 2016;203:647-652.

17. Dempsey JT, Matta LS, Carter DM, et al. Assessment of drug therapy-related issues in an outpatient heart failure population and the potential impact of pharmacist-driven intervention. $J$ Pharm Pract. 2017;30(3):318-323.

18. Stewart S, Pearson S, Horowitz JD. Effects of a home-based intervention among patients with congestive heart failure discharged from acute hospital care. Arch Intern Med. 1998;158(10):1067-1072.
19. Stewart S, Vandenbroek AJ, Pearson S, Horowitz JD. Prolonged beneficial effects of a home-based intervention on unplanned readmission and mortality among patients with congestive heart failure. Arch Intern Med. 1999;159(3):257-261.

20. Goodyer LI, Miskelly F, Milligan P. Does encouraging good compliance improve patients' clinical condition in heart failure? $\mathrm{Br} J$ Clin Pract. 1995;49(4):173-176.

21. Varma S, McElnay JC, Hughes CM, Passmore AP, Varma M. Pharmaceutical care of patients with congestive heart failure: interventions and outcomes. Pharmacotherapy. 1999;19(7):861-869.

22. Gattis WA, Hasselblad V, Whellan DJ, O'Connor CM. Reduction in heart failure events by the addition of a clinical pharmacist to a heart failure management team: results of the Pharmacist in Heart Failure Assessment Recommendation and Monitoring (PHARM) Study. Arch Intern Med. 1999;159(16):1939-1945.

23. Rainville EC. Impact of pharmacist interventions on hospital readmissions for heart failure. Am J Health Syst Pharm. 1999;56(13):1339-1342.

24. Whellan DJ, Gaulden L, Gattis WA, et al. The benefit of implementing a heart failure disease management program. Arch Intern Med. 2001;161(18):2223-2228

25. Patel KP, Sansgiry SS, Miller L. Pharmacist participation in home health heart failure programs. Am J Health Syst Pharm. 2003;60(21): $2259-2260$.

26. Lowrie R, Mair FS, Greenlaw N, et al; Heart Failure Optimal Outcomes from Pharmacy Study (HOOPS) Investigators. Pharmacist intervention in primary care to improve outcomes in patients with left ventricular systolic dysfunction. Eur Heart J. 2012;33(3):314-324.

27. Gwadry-Sridhar FH, Arnold JM, Zhang Y, Brown JE, Marchiori G, Guyatt G. Pilot study to determine the impact of a multidisciplinary educational intervention in patients hospitalized with heart failure. $\mathrm{Am}$ Heart J. 2005;150(5):982.

28. López Cabezas C, Falces Salvador C, Cubí Quadrada D, et al. Randomized clinical trial of a post-discharge pharmaceutical care program vs regular follow-up in patients with heart failure. Farm Hosp. 2006;30(6):328-342.

29. Jackevicius CA, de Leon NK, Lu L, Chang DS, Warner AL, Mody FV. Impact of a multidisciplinary heart failure post-hospitalization program on heart failure readmission rates. Ann Pharmacother. 2015;49(11):1189-1196.

30. Hale GM, Hassan SL, Hummel SL, Lewis C, Ratz D, Brenner M. Impact of a pharmacist-managed heart failure postdischarge (bridge) clinic for veterans. Ann Pharmacother. 2017;51(7):555-562.

31. Holland R, Battersby J, Harvey I, Lenaghan E, Smith J, Hay L. Systematic review of multidisciplinary interventions in heart failure. Heart. 2005;91:899-906.

32. Koshman SL, Charrois TL, Simpson SH, McAlister FA, Tsuyuki RT. Pharmacist care of patients with heart failure: a systematic review of randomized trials. Arch Intern Med. 2008;168(7):687-694.

33. Kang JE, Han NY, Oh JM, et al. Pharmacist-involved care for patients with heart failure and acute coronary syndrome: a systematic review with qualitative and quantitative meta-analysis. $J$ Clin Pharm Ther. 2016;41(2):145-157.

34. Jennings DL, Brewer R, Smith C, Williams C. Clinical pharmacist intervention for patients with left ventricular assist devices. Ann Pharmacother. 2011;45(10):1311-1312.

35. Ryder M, Murphy NF, McCaffrey D, O'Loughlin C, Ledwidge M, McDonald K. Outpatient intravenous diuretic therapy: potential for marked reduction in hospitalization for acute decompensated heart failure. Eur J Heart Fail. 2008;10(3):267-272.

36. Buckley LF, Carter DM, Matta L, et al. Intravenous diuretic therapy for the management of heart failure and volume overload in a multidisciplinary outpatient unit. JACC Heart Fail. 2016;4(1):1-8.

37. Milfred-LaForest SK, Chow SL, DiDomenico RJ, et al. Clinical pharmacy service in heart failure: an opinion paper from Heart Failure Society of America and American College of Clinical Pharmacy Cardiology Practice and Research Network. Pharmacotherapy. 2013;33(5):529-548. 
Integrated Pharmacy Research and Practice is an international, peer-reviewed, open access, online journal, publishing original research, reports, reviews and commentaries on all areas of academic and professional pharmacy practice. This journal aims to represent the academic output of pharmacists and pharmacy practice with particular focus on integrated care. All papers are carefully peer reviewed to ensure the highest standards as well as ensuring that we are informing and stimulating pharmaceutical professionals. The manuscript management system is completely online and includes a very quick and fair peer-review system, which is all easy to use. Visit http://www.dovepress.com/ testimonials.php to read real quotes from published authors.

Submit your manuscript here: http://www.dovepress.com/integrated-pharmacy-research-and-practice-journal 